Cross-validation of the Work Organization Assessment Questionnaire across genders: A study in the Australian Healthcare Sector

\author{
Leila Karimi, La Trobe University, Australia \\ Maria Karanika-Murray, Nottingham Trent University, UK \\ and Denny Meyer, Swinburne University of Technology, Australia
}

This is a non-final version of an article published in final form in:

Karimi, L., Karanika-Murray, M., \& Meyer, D. (accepted). Cross-validation of the Work

Organization Assessment Questionnaire across genders: A study in Australian healthcare organization. Journal of Occupational \& Environmental Medicine 


\begin{abstract}
Objectives The main aim of this study was to examine the measurement invariance of the Work Organisation Assessment Questionnaire (WOAQ) across genders in a group of healthcare employees, using bifactor modelling. There is a very limited research that uses invariance testing of bifactor models, despite their usefulness. Establishing validity of the WOAQ in this way is important for demonstrating its relevance for both males and females. Methods A bifactor modelling procedure was used here to examine the validity of the WOAQ with a sample of 946 paramedics employed in a large Australian organization in the healthcare sector.

Results The results of this study show that the WOAQ has good psychometric properties across genders in healthcare settings. In addition, there were significant mean differences between males and females in their perceptions of 'quality of relationships with colleagues', and 'reward and recognition'. There were no differences between males and females on the remaining factors: 'quality of relationships with the management', 'quality of relationships with colleagues', and 'quality of the physical environment'.
\end{abstract}

Conclusions The use of bifactor modelling to establish the cross-validity of the WOAQ across male and female paramedics adds to evidence for the measure's good psychometric properties. The findings add to those of previous research that has used higher order Confirmatory Factor Analysis (CFA). Moreover, mean differences between males and females were found to be significant in two of the five WOAQ subscales. These findings have practical implications for healthcare organizations, in terms of assessing work characteristics and developing activities to support the health and well-being of their employees. 


\section{Cross-validation of the Work Organization Assessment Questionnaire across genders:}

\section{A study in the Australian Healthcare Sector}

As the importance of work for employee health and well-being and the impact of employee health and well-being for the productivity of business organizations are increasingly being recognised, a better understanding of the relevant work characteristics has developed. Correspondingly, a number of instruments to assess work characteristics are currently available. Amongst these is the Work Organization Assessment Questionnaire (WOAQ), which offers a concise, reliable, and actionable solution for assessing psychosocial aspects of work and developing interventions to address these [1]. This study builds on existing on the psychometric properties of the WOAQ using bifactor modelling to establish the cross-validity of the measure in a sample of male and female paramedics.

The ability to reliably assess individuals' experience of work characteristics is especially important in high-risk sectors and occupations such as nursing. The nursing profession is especially prone to work-related ill-health [3-5]. A substantial body of evidence demonstrates the contribution of psychosocial working conditions to these issues [e.g., 6-7]. Accurate identification of the factors that can impact on health outcomes can inform the development of targeted interventions [1-2]. A key challenge is therefore to develop appropriate measurement tools that can accurately identify the most salient factors.

The WOAQ was initially developed as a sector-specific measure to help organizations in the manufacturing sector to identify the factors related to the design and management of work and to measure the impact of these factors on employee health and well-being [1]. The measure consists of 28 items that describe five aspects of work: 'quality of relationships with the management', 'reward and recognition', 'workload issues', 'quality of relationships with colleagues', and 'quality of the physical environment' [1]. A key requirement for measures that aim to assess suboptimal work characteristics is that they are 
relatively short but also comprehensive, that are easy to interpret by non-experts, and that provide sufficient feedback to employers to inform the development of action plans. The WOAQ meets these requirements: it has excellent reliability and validity properties and been used with a range of occupations in several countries [9]. For example, the WOAQ subscales have predicted well-being, subjective health, and job satisfaction [1], self-rated performance [2], as well as presenteeism, absenteeism, and turnover intentions [8].

Although originally developed for the manufacturing sector, the WOAQ has been validated and applied in a number of sectors, occupations, and workplace environments. For example, it has been utilised successfully in health care [9-11], the public sector $[28,8]$ and the financial sector [9]. Translated in Italian and validated against the GHQ12 and the Job Demands-Control model, it maintains its original characteristics [9]. The original study included manual, managerial, and office jobs in manufacturing and the WOAQ has proven to be broad enough to cover the core work characteristics covered, regardless of type of job or occupational sector. Although research has examined the WOAQ in a range of sectors and occupations, it has not yet examined its validity across genders.

Any workplace intervention for health and well-being should be beneficial for both males and females. 'Gender mainstreaming', or a gender-sensitive approach to workplace health, has been recognised as important by the European Commission in its community safety and health strategy 2002-06 [34]. Both groups of employees can better benefit from interventions if these are developed on the basis of a gender-sensitive approach. This starts from recognising possible gender differences in the work experience. Therefore, in recognition of the importance of a gender-sensitive approach to assessing work characteristics, this study will to examine whether males and females experience work characteristics differently, and in this way validate the WOAQ across males and females. Next we provide more detail on cross-validation and bifactor modelling. 


\section{A bifactor model of the WOAQ}

In a bifactor model (also referred to as direct hierarchical order modelling) all latent factors are modelled using first-order models that are nested within a general factor [12-14]. Despite its useability, bifactor modelling is not commonly applied in research [12, 15]. Using bifactor modelling to examine the validity of a widely-used measure such as the WOAQ is appropriate for two reasons. First, having a broad or macro-level assessment (using a general factor) would help to provide a solution for the whole organisation, while by assessing working conditions at a narrower or micro-level (using sub-factors) would have practical implications for the improvement of specific problematic areas. Thus, evaluating the plausibility of sub-factors is especially important for practice. Second, despite the appeal of the WOAQ for research and practice in non-manufacturing settings, establishing its latent structure is still ongoing, as highlighted in recent studies [2]. Therefore, a bifactor model will be used to provide further evidence on the validity of the WOAQ.

\section{Cross validity of bifactor model of WOAQ across genders}

An important aspect of a measure's psychometric properties is whether the measure fits well in different groups of target individuals or populations, i.e., its cross validity. Model invariance can be tested for two or more distinct samples using CFA. If the model fits well in all the samples then it is acceptable and valid across the corresponding populations. There are several procedures for evaluating cross-validity in CFA models, each relating to a hypothesis for a different set of key population parameters [16-20]. In this study, invariance testing will be conducted on a bifactor model of the WOAQ at both parameter and construct levels, building on recommendations for good practice [16, 17-20, 23, 25, 28].

In order to assess the validity and the cross-validity of the WOAQ, three types of analysis will be carried out across gender for the bifactor model WOAQ with its five nested factors. First, the validity of the bifactor model of WOAQ will be independently tested for 
male and female paramedics. Next, invariance testing for the measures will be carried out. Finally, if the model shows satisfactory invariance of the measures, the construct means will be tested for invariance.

\section{Method}

\section{Data collection and participants}

Data was originally collected from a large Australian health organisation employing paramedics. The study design was cross-sectional. An online self-report questionnaire was used to collect the data. In total, 979 responses were received from the paramedics. Of those, 33 were volunteer paramedics who were excluded from the final dataset. The final sample consisted of $n=X$ males and $n=Y$ females $(N=946)$. Human Research Ethics Committee approval for the study was originally obtained from the leading university.

\section{Measure}

The Work Organisation Assessment Questionnaire (WOAQ) [1] was used in this study. WOAQ consists of 28-items with a five-factor structure. The five-factor structure of the scale includes: workload issues, reward and recognition, quality of relationships with management, relationships with colleagues, and physical environment. Participants are asked to rate their experience with a number of aspects of work, on a 5-point Likert type scale (from 1=major problem to 5-very good).

\section{Data analysis}

A multisample bifactor model of confirmatory factor analysis (CFA) was conducted using EQS (version 6.2) to assess the cross validity of the WOAQ across male and female paramedics. At the first step, the baseline bifactor model (a nested construct where each item loads on a general construct and its nested construct) was tested separately for males and females. At the second step the cross validity of the WOAQ was assessed using invariance testing across genders. 
An important factor that was considered in choosing the suitable fit indices was the degree of penalty included for model complexity (please see Karimi \& Meyer, 2014 for full details). The fit indices reported in this study are the root mean square error of approximation (RMSEA), the Tucker-Lewis Index (TLI) or Non-normed fit index (NNFI), and the Akaike Information Criterion (AIC). RMSEA values lower than .08 and .05 [29] and NNFI values of greater than 0.90 and 0.95 [30] were considered as marginal and good fit, respectively. The model comparisons were performed based on changes in NNFI. NNFI differences of $\geq .010$ indicate significant model improvement [24]. The Akaike Information Criterion (AIC) is a comparative measure of fit. Smaller values of AIC demonstrate a superior model fit. The AIC increases of $>10$ indicate a significant deterioration in model fit [31-33]. In addition, the SB chi-square goodness of fit test and Comparative Fit Index (CFI) were also reported, being conventional and commonly used measures of fit in the literature.

\section{Results}

The demographics for the whole sample and separately for the male and female subsamples are presented in Table 1.

$$
\text { < insert Table } 1 \text { here > }
$$

At the first step, a baseline model (see Figure 1) was evaluated separately for both male and female samples. The results in Table 2 show adequate model fit for the baseline bifactor model for males $(\mathrm{RMSEA}=0.04, \mathrm{NNFI}=0.94)$ and females $(\mathrm{RMSEA}=0.05$, $\mathrm{NNFI}=0.92$ ).

\section{<insert Table 2 and Figure 1 here>}

Model 1: Configural model with no constraints. At the next step, a configural bifactor model was fitted for each group of male and female groups simultaneously to determine if the model is appropriate across gender when there are no constraints. The configural model showed good model fit across gender (RMSEA=0.03, NNFI=0.97; see Table 3). 
Model 2: Invariant loadings. After constraining the loadings to be equal for males and females, the results still showed good fit (RMSEA=0.03, NNFI=0.96). To test for evidence of invariance, the differences between the NNFI and AIC of Model 2 and Model 1 were considered. These suggested no significant deterioration in model fit for constrained loadings compared to the configural model of males and females (unconstrained loadings) in the case of NNFI in Table 3) but there was increase of $>10$ in the AIC.

As previously explained, reaching full invariance for all the parameters, or even the most important ones, is very rare in most models (e.g., Byrne, Shavelson \& Muthen, 1989). A decision was therefore made to proceed to the next stage of the invariance analysis considering the differences in construct means for males and females.

\section{<insert Table 3 here>}

Group Differences for Construct Mean. The differences of the general factor of WOAQ and the nested five factor means for male employees were examined with the female group means selected as the baseline. The construct means for females were set to zero while the construct means for males were estimated on the basis of the mean differences between groups for the constructs.

After setting equality constraints on loadings and intercepts for the measured variables, with factor intercepts of zero for female employees, the results showed an adequate fit to the model (RMSEA=0.05, NNFI=0.917). The mean differences between the male and female groups were significant on two of the nested constructs ('quality of support from colleagues' and 'reward and recognition') and also for the general factor of the WOAQ. The results indicated that the mean scores on these two nested constructs and the general factor of WOAQ were significantly higher for male than for female paramedics.

\section{Discussion}


The results of this study indicate that the WOAQ is a valid measurement tool to use with both male and female paramedics. Unlike previous studies that have used conventional second-order modelling using CFA, a bifactor model of WOAQ was cross-validated in this study. The results indicated that a bifactor model not only presents a good fit but also highlights the importance of the subscales relative to a single general factor in this setting. The results demonstrate good validity for the WOAQ across genders.

In addition, the mean differences between males and females were found to be significant. The scores on two of the five nested subfactors and the general WOAQ factor. These were significantly higher for male employees compared to female employees, demonstrating that male paramedics are happier with the quality of relationships with their colleagues, with rewards and recognition, and with the general quality of working conditions as assessed by the WOAQ than female paramedics in this organisation.

The results have important implications in practice, and specifically in relation to assessing the psychosocial issues that can contribute to paramedics' workplace health and well-being and developing targeted interventions for different groups of employees. As mentioned, the WOAQ was originally developed for use in the manufacturing sector, validated with a number of jobs within this sector, and proven to be useful in a number of other occupational sectors including health organisations [11]. This study offers additional evidence for its potential for accurately assessing working conditions and from this assessment developing targeted interventions for different groups of employees.

In conclusion, this study adds to the evidence supporting the use of the WOAQ for both research and practice in a range of settings. It offers a good coverage of work characteristics relevance to a range of jobs and sectors and demonstrates the need to be sensitive to possible qualitative differences in perceptions by different groups of employees. 
Future research should continue to examine the WOAQ with other occupational groups and sectors, preferably using bifactor modelling. 


\section{References}

1 Griffiths, A., Cox, T., Karanika, M., Khan, S., \& Tomás, J.M. (2006). Work design and management in the manufacturing sector: Development and validation of the Work Organisation Assessment scale. Occupational \& Environmental Medicine, 63(10), 669-675. doi: 10.1136/oem.2005.02367

2 Wynne-Jones, G., Buck, R., Varnava, C.J., \& Main, C. (2011). Impacts on work performance; what matters 6 months on? Occupational Medicine, 61, 205-208.

3 Hasselhorn H-M, Muller B H, \& Tackenberg P (2005, July). NEXT Scientific Report. Retrieved 22 September 2015 from http://www.econbiz.de/archiv1/2008/53602_nurses_work_europe.pdf

4 D’Errico, A., Viotti, S., Baratti, A., Mottura, B., Barocelli, A.P., Tagna, M., Scmbelluri, B., Battaglino, P., \& Converso, D. (2013). Low back pain and associated presenteeism among hospital nursing staff. Journal of Occupational Health, 55, 276-283.

5 Johnson, S., Cooper, C., Cartwright, S., Donald, I., Taylor, P., Millet, C. (2005). The experience of work - related stress across occupations. Journal of Managerial Psychology, 20(2), $178-187$.

6 Boumans, N. P. G., de Jong, A. H. J., \& Vanderlinden, L. (2008). Dtemrinants of early retirement intentions among Belgian nurses. Journal of Advanced Nursing, 63(1), 6474.

7 Fochsen, G., Josephson, M., Hagberg, M., Toomingas, A., \& Lagerström, M. (2006). Predictors of leaving nursing care: a longitudinal study among Swedish nursing personnel. Occupational \& Environmental Medicine, 63(3), 198-201. doi:10.1136/oem.2005.021956

8 Buck, R., Varnava, A., Wynne-Jones, G., Phillips. C., Farewell, D., Porteous, C., Webb, K., Buttton, L., Cooper, L., \& Main, C. (2008). Health and well-being in work in Merthyr 
Tydfill: A biopsychosocial approach. Well-being in Work Stage 2: Final Report to the Wales Centre for Health and Welsh Assembly Government.

www.wellbeinginwork.org

9 Magnavita N, Mammi F, Roccia K, \& Vincenti F (2007). WOA: un questionnario per la valutazione dell' organizzazione del lavoro. Traduzione e validazione della versione italiana. [WOA: a questionnaire for the evaluation of work organization. Translation and validation of the Italian version]. Giornale Italiano di Medicina del Lavoro ed Ergonomia, 29, 663-665.

10 Copertano, A., Bevilacqua, G., Barbaresi, M., Barchiesi, F., \& Copertano, B. (2010). Work-related stress: Risk assessment in the local regional health service unit of Ancona [La valutazione dello stress lavoro-correlato nell'azienda sanitaria di Ancona]. Giornale Italiano di Medicina del Lavoro ed Ergonomia, 29(4), 128-129.

11 Karimi, L., \& Meyer, D. (2014). Validity and Model-Based Reliability of the Work Organisation Assessment Questionnaire (WOAQ) Among Nurses. Nursing Outlook, $63(3)$.

12 Gignac, G. E. (2007). Multifactor modeling in individual differences research: Some recommendations and suggestions. Personality \& Individual Differences, 42, 37-48.

13 Gustafsson, J. E., \& Balke, G. (1993). General and specific abilities as predictors of school achievement. Multivariate Behavioral Research, 28, 407-434.

14 Holzinger, K. J., \& Swineford, F. (1937). The bi-factor method. Psychometrika, 2, 41-54. 15 Reise, P., Moore, T. M. and Haviland, M. G. (2010). Bifactor Models and Rotations: Exploring the Extent to which Multidimensional Data Yield Univocal Scale Scores. Journal of Personality Assessment, 92(6), 544-559.

16 Meredith,W. (1993). Measurement invariance, factor analysis and factorial invariance. Psychometrika, 58, 525-543. 
17 Widaman, K. F., \& Reise, S. P. (1997). Exploring the measurement invariance of psychological instruments: Applications in the substance use domain. In K. J. Bryant, M. Windle, \& S. G. West (Eds.), The science of prevention: Methodological advances from alcohol and substance abuse research (pp. 281-324). Washington, DC: American Psychological Association.

18 Byrne, B. M. (2006). Structural equation modelling with EQS: Basic concepts, application, and programming. New Jersey: Lawrence Elbaum Associates.

19 Byrne, B. M. and Watkins, D. (2003). The Issue of Measurement Invariance Revisited. Journal of Cross-Cultural Psychology, 34, 155-175.

20 Cheung, G. W. \& Rensvold, R. B. (2002): Evaluating Goodness-of-Fit Indexes for Testing Measurement Invariance. Structural Equation Modelling: A Multidisciplinary Journal, 9(2), 233-255.

21 Little, T. D. (1997). Mean and covariance structures (MACS) analyses of cross-cultural data: Practical and theoretical issues. Multivariate Behavioral Research, 32, 53-76.

22 Sörbom, D. (1974). A general method for studying differences in factor means and factor structures between groups. British Journal of Mathematical \& Statistical Psychology, 27, 229-239.

23 Chen, F. F., Sousa, K. H. \& West, S. G.(2005). Testing Measurement Invariance of Second-Order Factor Models. Structural Equation Modelling, 12(3), 471-492.

24 Vandenberg, R. J., \& Lance, C. E. (2000). A review and synthesis of the measurement invariance literature: Suggestions, practices, and recommendations for organisational research. Organisational Research Methods, 3, 4-69.

25 Meredith, W., \& Horn, J. (2001). The role of factorial invariance in modelling growth and change. In A. Sayer \& L. Collins (Eds.), New methods for the analysis of change (pp. 203-240). Washington, DC: American Psychological Association. 
26 Byrne, B. M., \& Campbell, T. L. (1999). Cross-cultural comparisons and the presumption of equivalent measurement and theoretical structure: A look beneath the surface. Journal of Cross-Cultural Psychology, 30, 555-574.

27 Marsh, H. W., \& Hocevar, D. (1985). Application of confirmatory factor analysis to the study of self-concept: First- and higher-order factor models and their invariance across groups. Psychological Bulletin, 97, 562-582.

28 Wynne-Jones, G., Varnava, A., Buck, R., Karanika-Murray, M., Griffiths, A., Phillips, C., \& Main, C. (2009). Examination of the Work Organisation Assessment Questionnaire in public sector workers. Journal of Occupational \& Environmental Medicine, 51(5), 586-593. doi: 10.1097/JOM.0b013e31819e9b

29 MacCallum, R. C., Browne, M. W., \& Sugawara, H. M. (1996). Power analysis and determination of sample size for covariance structure modeling. Psychological Methods, 1, 130-149.

$30 \mathrm{Hu}, \mathrm{L} .$, \& Bentler, P. M. (1999). Cutoff criteria for fit indexes in covariance structure analysis: Conventional criteria versus new alternatives. Structural Equation Modeling, $6,1-55$.

31 Akaike, H. (1973). Information theory and an extension of the maximum likelihood principle. In B. N. Petrov \& B. F. Csaki (Eds.), Second International Symposium on Information Theory, (pp. 267-281). Academiai Kiado: Budapest.

32 Raftery, Adrian E. 1995. Bayesian Model Selection in Social Research. Sociological Methodology, 25, 111-95.

33 Schwarz, G. (1978). Estimating the dimension of a model. Annals of Statistics, 6, 461-464.

34 EU-OSHA - European Agency for Safety and Health at Work (2014). Mainstreaming gender into occupational safety and health practice. Luxembourg: Office for Official Publications of the European Communities. 
Figure 1. The proposed baseline bifactor model of WOAQ 